\title{
What Would Be the Most Appropriate $\alpha / \beta$ Ratio in the Setting of Stereotactic Body Radiation Therapy for Early Stage Non-Small Cell Lung Cancer
}

\author{
Alexander Chi, ${ }^{1}$ Sijin Wen, ${ }^{2}$ Zhongxing Liao, ${ }^{3}$ Jack Fowler, ${ }^{4}$ Jiahong Xu, ${ }^{5}$ Nam P. Nguyen, \\ James S. Welsh, ${ }^{7,8}$ and Ritsuko Komaki ${ }^{3}$ \\ ${ }^{1}$ Department of Radiation Oncology, West Virginia University, Medical Center Dr., Morgantown, WV 26506, USA \\ ${ }^{2}$ Biostatistics Core, Mary Babb Randolph Cancer Center, West Virginia University, Morgantown, WV 26506, USA \\ ${ }^{3}$ Department of Radiation Oncology, The University of Texas MD Anderson Cancer Center, Houston, TX 77030, USA \\ ${ }^{4}$ Department of Human Oncology, University of Wisconsin, Madison, WI 53792, USA \\ ${ }^{5}$ Westat-An Employee-Owned Research Corporation, Rockville, MD 20850, USA \\ ${ }^{6}$ Department of Radiation Oncology, University of Arizona, Tucson, AZ 85724, USA \\ ${ }^{7}$ Department of Medical Physics, University of Wisconsin, Madison, WI 53792, USA \\ ${ }^{8}$ Department of Physics, Northern Illinois University, DeKalb, IL 60115, USA
}

Correspondence should be addressed to Alexander Chi; achiaz2010@gmail.com

Received 8 May 2013; Accepted 25 September 2013

Academic Editor: Rumiana Koynova

Copyright (C) 2013 Alexander Chi et al. This is an open access article distributed under the Creative Commons Attribution License, which permits unrestricted use, distribution, and reproduction in any medium, provided the original work is properly cited.

\begin{abstract}
We hypothesize that the correlation between the radiation dose expressed as the biologically effective dose (BED) and the clinical endpoints will correlate better as the value of the $\alpha / \beta$ ratio is increased to $>10 \mathrm{~Gy}$, which theoretically minimizes the overestimation of the dose potency associated with the linear quadratic (LQ) formula in the setting of stereotactic body radiation therapy (SBRT) for early stage non-small cell lung cancer (NSCLC). A search was conducted in the PubMed electronic databases in August 2011. In the studies analyzed, increasing the $\alpha / \beta$ ratio is associated with an increase in the strength of the correlation between isocenter BED and local control, especially in the studies with median followup of $\geq 24$ months, for which Spearman's correlation coefficients of 0.74 0.76 were achieved for $\alpha / \beta$ of $20 \mathrm{~Gy}, 30 \mathrm{~Gy}$, and $50 \mathrm{~Gy}(P=0.007-0.008)$. A trend toward statistical significance was observed for the correlation of isocenter BED and the 2 -year overall survival when an $\alpha / \beta$ of 20 Gy was used approached statistical significance $(P=0.073)$. Our results suggest that an $\alpha / \beta>10$ Gy may be more appropriate for the prediction of dose response in the setting of lung SBRT.
\end{abstract}

\section{Introduction}

Stereotactic body radiation therapy (SBRT), a technique which delivers an ablative dose of radiation over a short period of time, has emerged to become a major noninvasive treatment modality for early stage nonsmall cell lung cancer (NSCLC) worldwide. Excellent local control with tolerable toxicity profile has been consistently reported [1]. In operable patients with stage I NSCLC, long-term overall survival after SBRT may be at least comparable to that after surgery when an adequate dose of radiation was delivered [2]. These excellent clinical outcomes provide evidence for SBRT's increasing role in the treatment of early stage NSCLC as an alternative to surgery.

Due to the large variation in the dose fractionation schedules used in clinical practice, dose response following SBRT was investigated and demonstrated after the radiation dose delivered is converted to the biologically effective dose (BED) after linear quadratic normalization $[3,4]$. The BED, the total dose which could cause the same log cell kill as a specific dose fractionation schedule under consideration if it is delivered in infinitely small fractions well spaced out or at an infinitely low-dose rate, is a mathematical term derived from linear quadratic cell survival outcome in radiobiology 
[5]. Because of the short overall treatment time (usually $<2$ weeks), the overall treatment time factor is often ignored in the BED calculation when SBRT was delivered for early stage NSCLC [3-5].

Despite this wide adaptation of the BED in lung SBRT, many have questioned its validity because of the potential overestimation of tumor cell kill when large doses of radiation are delivered in 1 fraction by the linear quadratic (LQ) formalism [6-8]. To avoid overpredicting the potency and toxicity of SBRT, a new mathematical model, the universal survival curve (USC), has been created to better fit the NSCLC cell survival curve [9]. This model was created by adopting the multitarget model of tumor cell kill (a model which predicts cell survival by assuming that cell kill depends on whether a number of critical targets within a cell are hit after irradiation) into the LQ formalism at a certain radiation dose level, the transition dose, because the multitarget model has been shown to fit empirical cell survival data well in the high-dose range. Although being very sophisticated, the complexity of this model poses a challenge to the clinician in day to day clinical practice. In addition, there are a number of parameters which need to be defined and validated prior to further clinical adaptation. On the contrary, the LQ model has been used for decades in the radiation oncology community and is widely adapted to clinical use. Therefore, simpler approaches to minimizing the overestimation of tumor cell kill after ablative doses of radiation through the LQ model should be sought.

As shown previously, NSCLC cells can possibly repopulate as fast as oropharyngeal cancer cells [10]. It has been observed that rapidly repopulating tumors are likely to have a greater $\alpha / \beta$ ratio, which is approximately $20 \mathrm{~Gy}$ for oropharyngeal cancers [11]. Therefore, a tumor $\alpha / \beta$ ratio of higher than 10 Gy may be more appropriate for NSCLC. Higher $\alpha / \beta$ ratio has been known to produce straighter curves in the high-dose region through the LQ model. Therefore, the potential overestimation of dose response in high-dose regions by the LQ model in the setting of SBRT for early stage NSCLC may be reduced by simply using an $\alpha / \beta$ ratio of higher than 10 Gy when calculating tumor BED, especially when large fractional doses are used [12]. In the current study, we investigate the dose response relationship between the tumor isocenter BED and local tumor control following SBRT for early stage NSCLC and explore which $\alpha / \beta$ ratio is the most appropriate for tumor BED calculation in this setting.

\section{Materials and Methods}

2.1. Search Strategy. This systematic review was designed to explore what $\alpha / \beta$ ratio should be most appropriate for BED calculation in the setting of SBRT for early stage NSCLC. This is assessed by investigating the strength of correlation between the tumor BED at the isocenter of the target calculated with various randomly selected $\alpha / \beta$ ratios $(5,8.2$, $10,20,30$, and $50 \mathrm{~Gy}$ ) and the clinical endpoints of local tumor control and overall survival. A search based on PubMed electronic databases was conducted to select studies outlining the following: local control for early stage NSCLC following
SBRT; toxicity following SBRT for NSCLC. The following terms were explored and used for each database search: non-small cell lung cancer, early stage, stage I, stereotactic body radiotherapy, stereotactic radiosurgery, and stereotactic ablative radiotherapy. Reference lists of relevant papers were then searched for additional publications. The tumor BED was calculated based on the linear quadratic formula with $\alpha / \beta$ ratios described above. For this study, the tumor BED is the BED at the isocenter of the target volume. Local control refers to the rate of tumor control at the primary site only for the duration of each study. It equals the number of patients with the tumor controlled locally decided by the total number of patients treated for a specific dose fractionation schedule.

2.2. Statistical Analysis. Scatterplots and nonparametric regression lines are generated using lowess smoothers $[13,14]$ to illustrate the relationship between radiation dose and clinical endpoints. A nonparametric Spearman's rank correlation coefficient [15] was calculated to estimate the degree of correlation between the radiation dose, which is expressed as the tumor BED at the target volume's isocenter, and the local control and the 2-year overall survival rates obtained from different studies, assuming that the relationship between the tumor BED and these clinical endpoints can be described by a nonlinear monotonic function.

\section{Results}

3.1. Quantity of Studies. A total of 24 studies reporting the clinical outcome associated with one dose fractionation scheme for which the isocenter dose can be calculated (most commonly used scheme if outcome following multiple dose fractionation schemes was reported) following SBRT delivered with photon therapy for stage I, and mostly T1, NSCLC were identified [16-39]. They were found through the PubMed electronic database searches after exclusion of duplicate, irrelevant references, as well as abstracts. All studies were published between 2003 and 2011. The studies used for the current analysis include most retrospective and a few prospective studies $[16,20-22,24,26,35]$. The most commonly used dose fractionation schedule, the number of patients treated with the listed dose fractionation schedule/the total number of patients underwent SBRT reported in each study, median follow-up time in months for each study, the associated local tumor control from each study, the dose calculation algorithm, and whether image guidance was used for radiation delivery are summarized in Table 1. The use of IGRT in the study by Fakiris et al. [16] was implied from a previous publication [40]. For the purpose of this study, studies of no more than 10 patients and the studies in which no description of the local tumor control for a specific dose fractionation regimen can be obtained in the actual publication or through communication with the corresponding authors were excluded. Also, studies that were published only in abstract form were excluded. For studies from the same group of investigators, only the latest publication detailing the specific dose fractionation regimen and corresponding local tumor control was included. 
TABLE 1: Local tumor control following SBRT for early stage NSCLC.

\begin{tabular}{|c|c|c|c|c|c|c|}
\hline References & No. of fractions & $\begin{array}{l}\text { No. of } \\
\text { patients/no. of } \\
\text { total patients }\end{array}$ & $\begin{array}{l}\text { Fractional dose } \\
\text { (isocenter, Gy) }\end{array}$ & $\begin{array}{l}\text { Median } \\
\text { followup } \\
\text { (months) }\end{array}$ & $\begin{array}{l}\text { Local } \\
\text { control }\end{array}$ & $\begin{array}{l}\text { Dose calculation } \\
\text { algorithm }\end{array}$ \\
\hline Kopek et al. [17] & 3 & $62 / 88$ & 15.0 & 44.0 & $93.1 \%$ & $\mathrm{~PB}^{9}$ \\
\hline Koto et al. [22] & & $20 / 31$ & 15.0 & 32.0 & $75.0 \%$ & Bathos \\
\hline Hoyer et al. [24] & & $40 / 40$ & 15.0 & 29.0 & $92.5 \%$ & $\mathrm{NSS}^{9}$ \\
\hline Bral et al. [35] & & $23 / 40$ & 18.0 & 16.0 & $91.3 \%$ & NSS, BrainLabs \\
\hline Ricardi et al. [26] & & $62 / 62$ & 18.8 & 28.0 & $93.6 \%$ & $\mathrm{CC}^{9}$ \\
\hline Guckenberger et al. [37] & & $32 / 124$ & 19.2 & 14.0 & $100.0 \%$ & $\mathrm{CC}^{\mathrm{g}}$ \\
\hline Ng et al. [30] & & $12 / 20$ & 20.0 & 21.0 & $83.3 \%$ & NSS, BrainLab \\
\hline Andratschke et al. [31] & & $92 / 92$ & $20.8^{*}$ & 21.0 & $89.1 \%$ & NSS \\
\hline Nyman et al. [18] & & $45 / 45$ & 21.0 & 43.0 & $80.0 \%$ & Bathos \\
\hline Vahdat et al. [19] & & $20 / 20$ & $21.3^{*}$ & 43.0 & $95.0 \%$ & $\begin{array}{c}\text { NSS, } \\
\text { Cyberknife }\end{array}$ \\
\hline Crabtree et al. [33] & & $76 / 76$ & 21.8 & 19.0 & $93.4 \%$ & NSS, Triology \\
\hline Baumann et al. [20] & & $57 / 57$ & 22.0 & 35.0 & $93.0 \%$ & $\mathrm{~PB}^{9}$ \\
\hline Timmerman et al. [21] & & $59 / 59$ & 22.5 & 34.4 & $98.2 \%$ & $\mathrm{NSS}^{9}$ \\
\hline Turzer et al. [38] & & $31 / 36$ & 22.9 & 13.8 & $100.0 \%$ & $\mathrm{CC}^{g}$ \\
\hline Fakiris et al. [16] & & $34 / 70$ & 25.0 & 50.2 & $97.1 \%$ & NSS \\
\hline van der Voort van Zyp et al. [36] & & $59 / 70$ & 25.8 & 15.0 & $96.6 \%$ & $\begin{array}{c}\text { NSS, } \\
\text { Cyberknife }\end{array}$ \\
\hline Matsuo et al. [23] & 4 & $101 / 101$ & 12 & 31.4 & $86.1 \%$ & PB \\
\hline Baba et al. [28] & & $85 / 124$ & 12 & 26.0 & $87.1 \%$ & NSS \\
\hline Stephans et al. [32] & 5 & $56 / 86$ & 10.2 & 19.8 & $96.4 \%$ & NSS, BrainLab \\
\hline Takeda et al. [29] & & $63 / 63$ & 12.5 & 24.0 & $95.6 \%$ & SC \\
\hline Lagerwaard et al. [25] & & $>100^{\ddagger} /-$ & 15.0 & 29.0 & $95.7 \%$ & NSS, BrainLab \\
\hline Onimaru et al. [34] & 8 to 10 & $17 / 45$ & 6.0 & 18.0 & $70.6 \%$ & PB \& SC \\
\hline Onishi et al. [39] & & $35 / 35$ & 7.3 & 13.0 & $94.3 \%$ & $\mathrm{NSS}^{9}$ \\
\hline Xia et al. [27] & & $43 / 43$ & 10.0 & 27.0 & $96.0 \%$ & $\begin{array}{l}\text { NSS, Gamma } \\
\text { knife }\end{array}$ \\
\hline
\end{tabular}

${ }^{*}$ Median or mean dose; ${ }^{\ddagger}$ Personal communication with Dr. Lagerwaard. 9 Image guidance applied. CC: Collapsed cone; NSS: not specifically stated; PB: pencil beam; S: superposition.

3.2. Correlation of Fractional Dose and Local Control. As shown in Table 1, a 3-fraction schedule delivering fractional doses from $15 \mathrm{~Gy}$ to $25.8 \mathrm{~Gy}$ to the tumor isocenter was used in 16/24 studies. A positive dose response between the fractional dose and local control among these studies has been observed with a Spearman's rank correlation coefficient of $0.55(P=0.033)$. This dose response relationship is shown in Figure 1. This dose response relationship was not observed in studies that used $\geq 4$ fractions most likely due to the small number of studies.

3.3. Correlation of Isocenter Tumor BED and Local Control. After the isocenter BEDs were calculated with gradually increasing but randomly selected values of $\alpha / \beta$ ratio through the linear quadratic formula, they were correlated with the associated local control values. As shown in Figure 2, increased isocenter BED correlated with an increase in local control regardless of which $\alpha / \beta$ ratio was used in the BED calculation. The isocenter BEDs calculated with $\alpha / \beta$ ratios

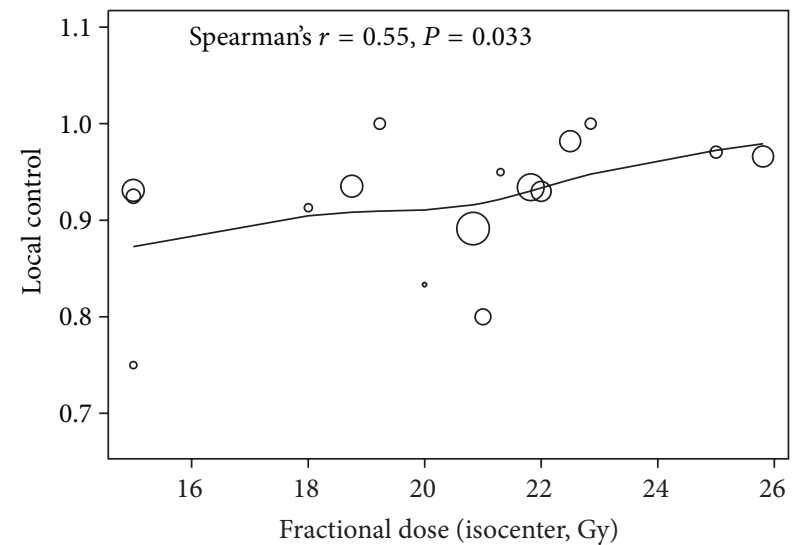

FIGURE 1: The correlation between the fractional dose and the local control in studies which reported the outcome of dose fractionation schedules that delivered the total dose in 3 fractions. The size of the circle is proportional to the number of patients treated with a specific dose fractionation schedule in each study. 


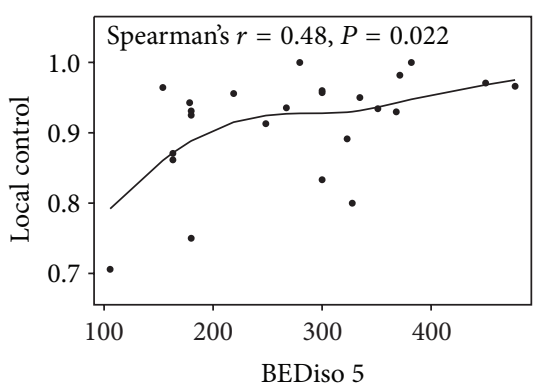

(a)

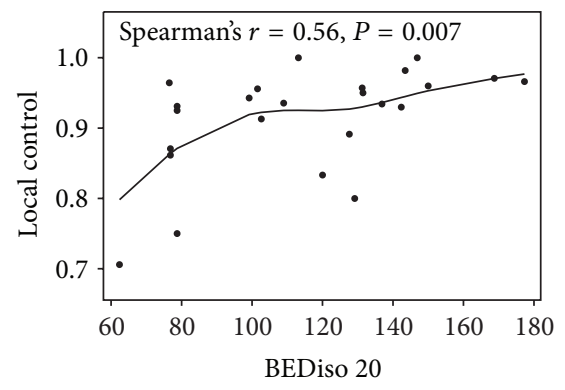

(d)

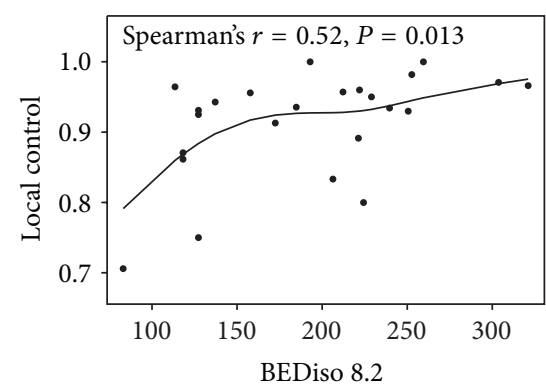

(b)

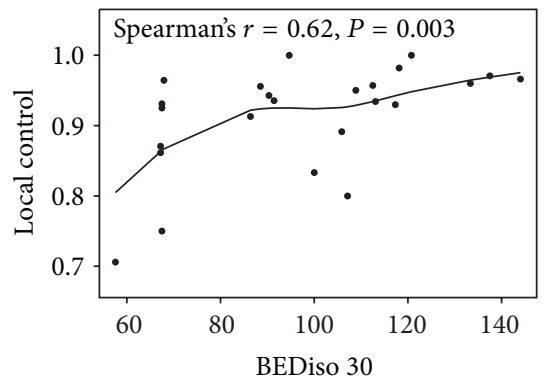

(e)

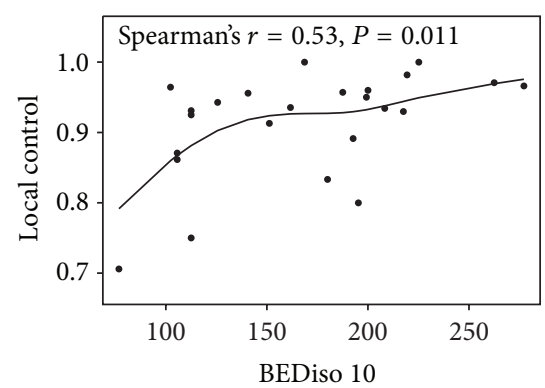

(c)

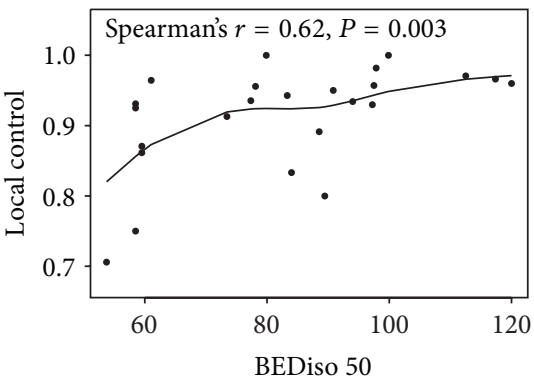

(f)

FIGURE 2: The correlation of the tumor BED at the isocenter calculated with a spectrum of $\alpha / \beta$ ratios $(5,8.2,10,20,30$, and 50 Gy) and the local control reported among all studies. Due to the use of different $\alpha / \beta$ ratios, the BED scale on the $x$-axis is different for each plot.

ranging from 5 to 50 Gy were all significantly correlated with the local control. However, the Spearman's rank correlation coefficient appeared to increase more noticeably as the value of $\alpha / \beta$ ratio increased to over $10 \mathrm{~Gy}$. The strength of correlation (statistical significance) between tumor isocenter $\mathrm{BED}$ and the local control also increased as the $\alpha / \beta$ ratio increased, especially when an $\alpha / \beta$ ratio of $>10$ Gy was used.

When studies with median followup of at least 24 months were analyzed separately [16-29], correlation between the isocenter $\mathrm{BED}$ calculated with the same set of $\alpha / \beta$ ratios and the local control rate reported in these studies was also observed. As shown in Figure 3, a trend toward stronger correlation between isocenter BED and local control is observed as higher $\alpha / \beta$ ratio is used in BED calculation. The highest correlation is observed for $\alpha / \beta$ ratios of 20,30 , and $50 \mathrm{~Gy}$ with correlation coefficients of $0.74-0.76$ (Figure 3 ).

\subsection{Correlation of Isocenter Tumor BED with 2-Year Overall} Survival. Seven of the 24 studies reported overall survival (OS) at 2 years [18-20, 24, 27, 29, 39]. Among all the survival endpoints reported, including disease-free survival, causespecific survival, and overall survival (OS), only OS at 2 years [18-20, 24, 27, 29, 39] demonstrated a positive correlation with radiation dose described as the tumor BED at the treatment isocenter. This is shown in Figure 4. The positive correlation is most noticeable when the isocenter BED is calculated with $\alpha / \beta$ ratios of $>10 \mathrm{~Gy}$. The strongest correlation was observed when the isocenter BED is calculated with an $\alpha / \beta$ ratio of $20 \mathrm{~Gy}$, and this correlation approached statistical significance with a Spearman's rank correlation coefficient of $0.75(P=0.073)$.

\section{Discussion}

As a tissue specific fractionation sensitivity parameter in the LQ formula, the $\alpha / \beta$ ratio has commonly been accepted to be approximately $10 \mathrm{~Gy}$ for tumor or "early responding" tissue. However, more studies are reporting a highly variable range of $\alpha / \beta$ ratios for various cancers based on individual tumor's rate of proliferation $[10,11,41,42]$. "Individualization" of $\alpha / \beta$ ratios based on specific tumor type may be important in the setting of SBRT, in which the LQ model has been criticized for overpredicting dose potency when large fractional dose, such as $20 \mathrm{~Gy}$, is delivered [9]. This is because of the fact that the overestimation of radiation efficacy can be effectively reduced if the $\alpha / \beta$ ratio is increased to $20 \mathrm{~Gy}$ to account for the rapidly proliferating nature of NSCLC cells when SBRT is used to deliver large fractions over $\leq 5$ fractions [12]. On the other hand, some have found that the LQ model may underestimate the tumor control after stereotactic radiosurgery intracranially, which may be caused by indirect tumor cell killing triggered by significant vascular damage after ablative doses are delivered [43]. However, this concept needs to be further explored in future studies on NSCLC.

The most important finding of this study is that the biologically effective dose response relationship in the setting of lung SBRT appears to be very stable throughout a spectrum of $\alpha / \beta$ ratios. This implies the robustness of the linearquadratic (LQ) formalism in predicting dose response and its clinical applicability in the setting of lung SBRT. The correlation between local control and the isocenter BED increased when the $\alpha / \beta$ ratio used in BED calculation increased from $5 \mathrm{~Gy}$ to $50 \mathrm{~Gy}$ (Figures 2 and 3). The best correlation was observed when studies with at least a median followup of 


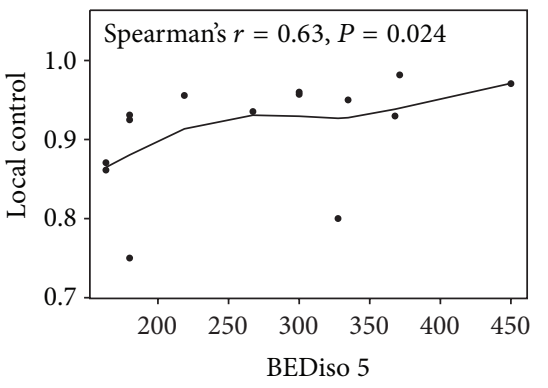

(a)

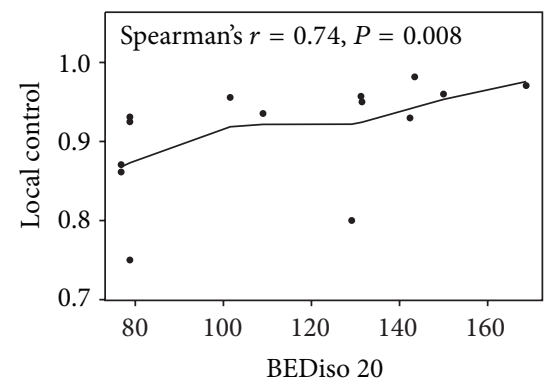

(d)

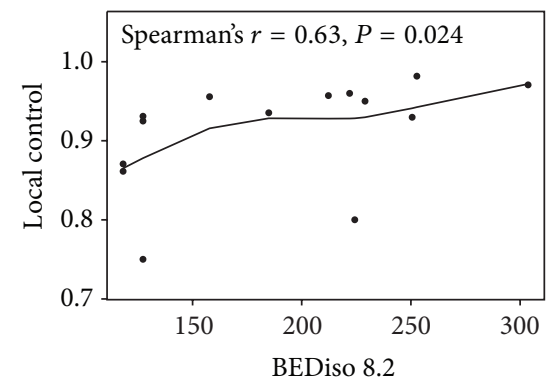

(b)

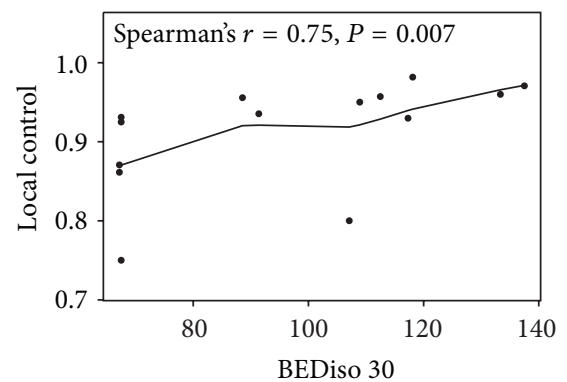

(e)

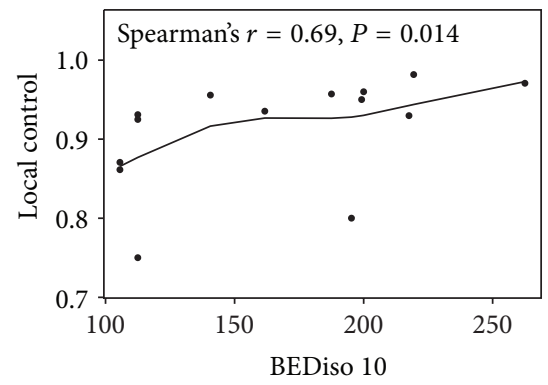

(c)

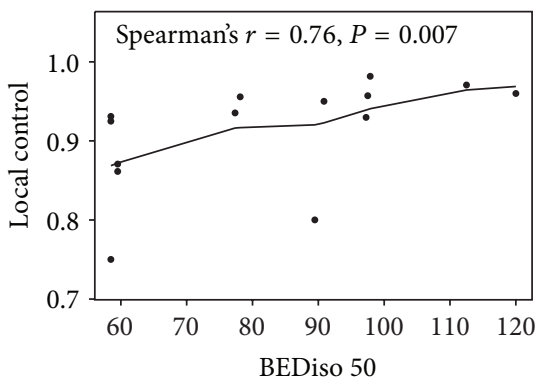

(f)

FIGURE 3: The correlation of the tumor BED at the isocenter calculated with a spectrum of $\alpha / \beta$ ratios $(5,8.2,10,20,30$, and $50 \mathrm{~Gy})$ and the local control reported among studies with a median followup of $\geq 24$ months. Due to the use of different $\alpha / \beta$ ratios, the BED scale on the $x$-axis is different for each plot.

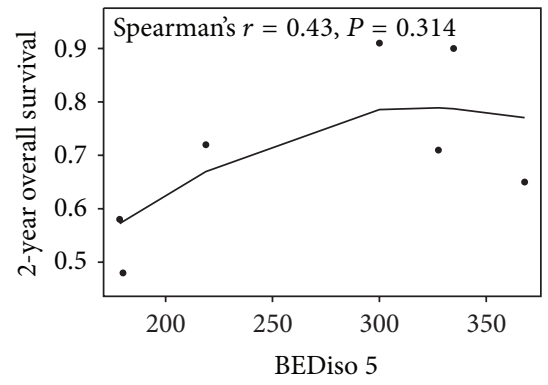

(a)

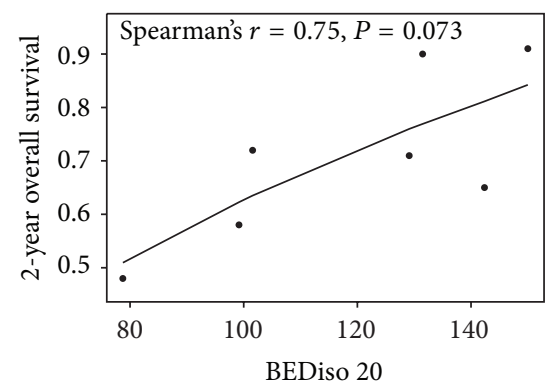

(d)

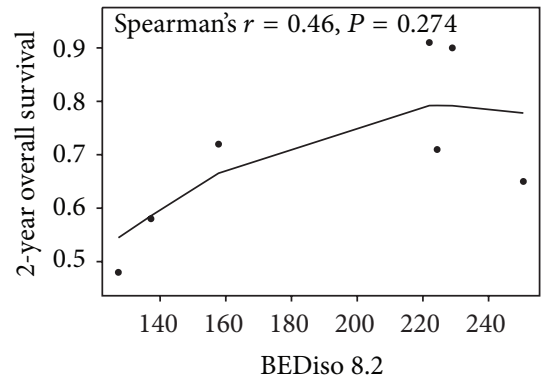

(b)

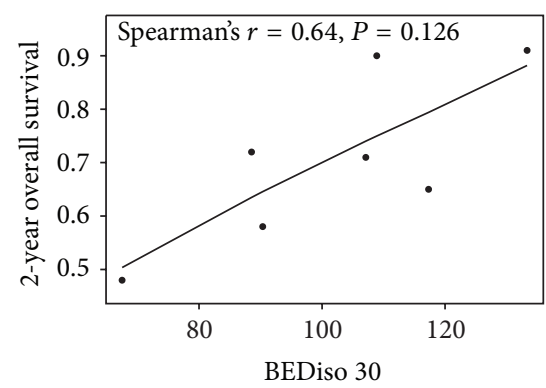

(e)

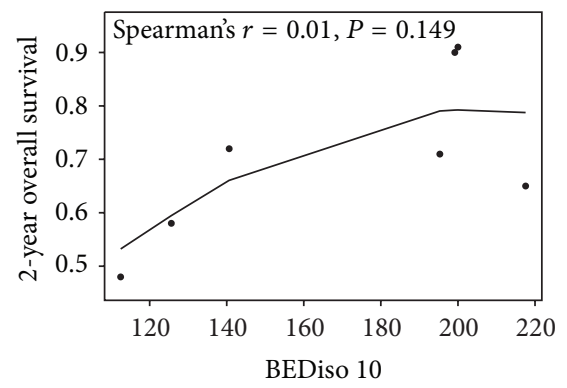

(c)

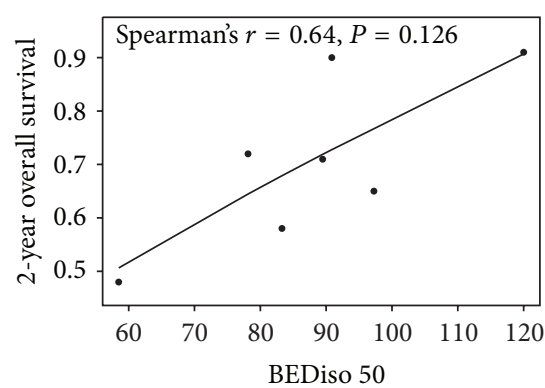

(f)

FIGURE 4: The correlation of the tumor BED at the isocenter calculated with a spectrum of $\alpha / \beta$ ratios $(5,8.2,10,20,30$, and $50 \mathrm{~Gy})$ and the 2 -year overall survival reported in selected studies. Due to the use of different $\alpha / \beta$ ratios, the BED scale on the $x$-axis is different for each plot. 
24 months were analyzed separately (Figure 3 ). These studies were analyzed separately because longer followup usually brings more stability and reliability to the results reported. Thus, the clinical evidence suggests that utilizing an $\alpha / \beta$ ratio of $>10$ Gy in the LQ formalism may lead to more accurate description of the biologically effective dose response relationship in the setting of SBRT for early stage NSCLC. Among all the survival data endpoints, the OS at 2 years among 7 studies also demonstrated a correlation with the BED. Within the spectrum of $\alpha / \beta$ ratios tested, this correlation was the strongest with a Spearman's correlation coefficient of 0.75 when an $\alpha / \beta$ ratio of $20 \mathrm{~Gy}$ was used for BED calculation (Figure 4). This correlation reached marginal statistical significance $(P=0.073)$. This finding supports the use of higher $\alpha / \beta$ ratios, such as $20 \mathrm{~Gy}$, for the prediction of biologically effective dose response in the setting of lung SBRT. For an $\alpha / \beta$ ratio of infinity, the Spearman's correlation coefficient was $0.40(P=0.15)$ for correlation between local control and tumor BED in studies with median followup of at least 24 months. Similarly, there was no statistically significant correlation when physical dose was used to correlate with 2 -year OS $(\varrho=0.02 ; P=0.99$ ). This suggests that the physical dose should not be used directly for dose response estimation in the setting of lung SBRT.

The robustness of the LQ model in the prediction of biologically effective dose response observed in our study is also supported by other studies $[44,45]$. The value of the $\alpha / \beta$ ratio to be used for iso-dose effect comparison of the SBRT dose fractionation schedules and conventionally fractionated dose regimens has been estimated to be $8.2 \mathrm{~Gy}$ in a modeling study by Stuschke and Pöttgen [44]. In another modeling study by Partridge et al., the dose response relationship between the tumor BED and the disease-free survival (DFS) for stage I-III NSCLC treated with both conventionally fractionated radiotherapy and SBRT has been explored [45]. The fitting of their dose response model appears to be very stable with $\alpha / \beta$ ranging from $8 \mathrm{~Gy}$ to $14 \mathrm{~Gy}$. Our study is different from the previous studies in that it further suggests that an $\alpha / \beta$ of $>10$ Gy may be comparatively more appropriate in the setting of lung SBRT even that spectra of $\alpha / \beta$ ratios from $5 \mathrm{~Gy}$ to $50 \mathrm{~Gy}$ may all be valid. However, this should be further validated in a large prospective study to include NSCLC patients who are treated with both conventionally fractionated radiotherapy and SBRT. This will need to be conducted to further investigate if a single $\alpha / \beta$ that would be suitable for both conventionally fractionated radiotherapy and SBRT can be defined. Furthermore, our study analyzes a relatively more homogeneous group of studies which report the clinical outcome following SBRT only. This minimizes the confounding factors, such as the delayed tumor cell repopulation associated with conventionally fractionated radiotherapy, the difference in reassortment, and reoxygenation between conventionally fractionated radiotherapy and ablative radiotherapy, as well as differences in treatment outcome related to the different technology used for radiotherapy delivery.

Spearman's correlation coefficient was used to assess the relationship between the tumor BED and the clinical endpoints assuming a nonlinear monotonic function. However, the study size was not taken into account when Spearman's correlation coefficient was calculated, which is a major limitation of our study. As a result, the findings from this study can only be suggestive and need to be further validated in future studies. Also, the heterogeneous nature of the different patient populations included in different studies greatly limits the study and warrants future investigation in a study that will assess tumor BED and clinical outcome based on individual patient outcome. Another limitation is that there were only 7 studies reporting the 2-year overall survival, which may be too few to establish a correlation with a high level of confidence.

\section{Conclusion}

Our study demonstrates that increasing the $\alpha / \beta$ ratio is associated with an increasing trend in the strength of the biologically effective dose response relationship, especially when the $\alpha / \beta$ ratio is increased to $\geq 20 \mathrm{~Gy}$. All of our findings support the previous hypothesis that the overestimation of tumor cell kill from the LQ model may be reduced by simply increasing the value of $\alpha / \beta$ [12]. However, our study is limited by the heterogeneous nature of different studies. As a result, this study cannot provide a definite $\alpha / \beta$ ratio in the setting of SBRT for early stage NSCLC. But it does suggest that an $\alpha / \beta>10$ Gy may be more appropriate for the prediction of dose response in the setting of lung SBRT.

\section{Conflict of Interests}

The authors declare that they have no conflict of interests.

\section{Acknowledgments}

The authors acknowledge the help and guidance from Drs. Frank J. Lagerwaard, Department of Radiation Oncology, VU University Medical Center, Amsterdam, The Netherlands; Yongshun Chen and You Lu MD, Division of Thoracic Cancer, Huaxi Hospital, Sichuan University, Chengdu, China; and Dr. Fumiya Baba, Department of Radiology, Nagoya City University Graduate School of Medical Sciences, Nagoya, Japan.

\section{References}

[1] A. Chi, Z. Liao, N. P. Nguyen, J. Xu, B. Stea, and R. Komaki, "Systemic review of the patterns of failure following stereotactic body radiation therapy in early-stage non-small-cell lung cancer: clinical implications," Radiotherapy and Oncology, vol. 94, no. 1, pp. 1-11, 2010.

[2] H. Onishi, H. Shirato, Y. Nagata et al., "Stereotactic body radiotherapy (SBRT) for operable Stage I non-small-cell lung cancer: can SBRT be comparable to surgery?" International Journal of Radiation Oncology Biology Physics, vol. 81, no. 5, pp. 13521358, 2011.

[3] H. Onishi, H. Shirato, Y. Nagata et al., "Hypofractionated stereotactic radiotherapy (HypoFXSRT) for stage I non-small cell lung cancer: updated results of 257 patients in a Japanese 
multi-institutional study," Journal of Thoracic Oncology, vol. 2, supplement 3, no. 7, pp. S94-S100, 2007.

[4] J. Wulf, K. Baier, G. Mueller, and M. P. Flentje, "Dose-response in stereotactic irradiation of lung tumors," Radiotherapy and Oncology, vol. 77, no. 1, pp. 83-87, 2005.

[5] J. F. Fowler, "21 Years of biologically effective dose," British Journal of Radiology, vol. 83, no. 991, pp. 554-568, 2010.

[6] M. Guerrero and X. A. Li, "Extending the linear-quadratic model for large fraction doses pertinent to stereotactic radiotherapy," Physics in Medicine and Biology, vol. 49, no. 20, pp. 4825-4835, 2004.

[7] H. Iwata, Y. Shibamoto, R. Murata et al., "Estimation of errors associated with use of linear-quadratic formalism for evaluation of biologic equivalence between single and hypofractionated radiation doses: an in vitro study," International Journal of Radiation Oncology Biology Physics, vol. 75, no. 2, pp. 482-488, 2009.

[8] R. Timmerman, M. Bastasch, D. Saha, R. Abdulrahman, W. Hittson, and M. Story, "Optimizing dose and fractionation for stereotactic body radiation therapy: normal tissue and tumor control effects with large dose per fraction," Frontiers of Radiation Therapy and Oncology, vol. 40, pp. 352-365, 2011.

[9] C. Park, L. Papiez, S. Zhang, M. Story, and R. D. Timmerman, "Universal survival curve and single fraction equivalent dose: useful tools in understanding potency of ablative radiotherapy," International Journal of Radiation Oncology Biology Physics, vol. 70, no. 3, pp. 847-852, 2008.

[10] J. F. Fowler and R. Chappell, "Non-small cell lung tumors repopulate rapidly during radiation therapy," International Journal of Radiation Oncology Biology Physics, vol. 46, no. 2, pp. 516-517, 2000.

[11] B. Maciejewski, H. R. Withers, J. M. G. Taylor, and A. Hliniak, "Dose fractionation and regeneration in radiotherapy for cancer of the oral cavity and oropharynx: tumor dose-response and repopulation," International Journal of Radiation Oncology Biology Physics, vol. 16, no. 3, pp. 831-843, 1989.

[12] J. F. Fowler, "Linear quadratics is alive and well: in regard to park et al. (Int J Radiat Oncol Biol Phys 2008;70:847-852)," International Journal of Radiation Oncology Biology Physics, vol. 72, no. 3, p. 957, 2008.

[13] W. S. Cleveland, "Robust locally weighted regression and smoothing scatterplots," The American Statistical Association, vol. 74, no. 368, pp. 829-836, 1979.

[14] W. S. Cleveland and S. J. Devlin, "Locally weighted regression: an approach to regression analysis by local fitting," The American Statistical Association, vol. 83, no. 403, pp. 596-610, 1988.

[15] J. Neter, M. H. Kutner, and W. Wasserman, Applied Linear Regression Models, Richard D Irwin, 1989.

[16] A. J. Fakiris, R. C. McGarry, C. T. Yiannoutsos et al., "Stereotactic body radiation therapy for early-stage non-small-cell lung carcinoma: four-year results of a prospective phase II study," International Journal of Radiation Oncology Biology Physics, vol. 75, no. 3, pp. 677-682, 2009.

[17] N. Kopek, M. Paludan, J. Petersen, A. T. Hansen, C. Grau, and M. Høyer, "Co-morbidity index predicts for mortality after stereotactic body radiotherapy for medically inoperable earlystage non-small cell lung cancer," Radiotherapy and Oncology, vol. 93, no. 3, pp. 402-407, 2009.

[18] J. Nyman, K.-A. Johansson, and U. Hultén, "Stereotactic hypofractionated radiotherapy for stage I non-small cell lung cancer: mature results for medically inoperable patients," Lung Cancer, vol. 51, no. 1, pp. 97-103, 2006.
[19] S. Vahdat, E. K. Oermann, S. P. Collins et al., "CyberKnife radiosurgery for inoperable stage IA non-small cell lung cancer: $18 \mathrm{~F}$ fluorodeoxyglucose positron emission tomography/computed tomography serial tumor response assessment," Journal of Hematology and Oncology, vol. 3, article 6, 2010.

[20] P. Baumann, J. Nyman, M. Hoyer et al., "Outcome in a prospective phase II trial of medically inoperable stage I nonsmall-cell lung cancer patients treated with stereotactic body radiotherapy," Journal of Clinical Oncology, vol. 27, no. 20, pp. 3290-3296, 2009.

[21] R. Timmerman, R. Paulus, J. Galvin et al., "Stereotactic body radiation therapy for inoperable early stage lung cancer," Journal of the American Medical Association, vol. 303, no. 11, pp. 10701076, 2010.

[22] M. Koto, Y. Takai, Y. Ogawa et al., "A phase II study on stereotactic body radiotherapy for stage I non-small cell lung cancer," Radiotherapy and Oncology, vol. 85, no. 3, pp. 429-434, 2007.

[23] Y. Matsuo, K. Shibuya, Y. Nagata et al., "Prognostic factors in stereotactic body radiotherapy for non-small-cell lung cancer," International Journal of Radiation Oncology Biology Physics, vol. 79, no. 4, pp. 1104-1111, 2011.

[24] M. Hoyer, H. Roed, A. T. Hansen et al., "Prospective study on stereotactic radiotherapy of limited-stage non-small-cell lung cancer,' International Journal of Radiation Oncology Biology Physics, vol. 66, no. 4, supplement, pp. S128-S135, 2006.

[25] F. J. Lagerwaard, C. J. A. Haasbeek, E. F. Smit, B. J. Slotman, and S. Senan, "Outcomes of risk-adapted fractionated stereotactic radiotherapy for stage I non-small-cell lung cancer," International Journal of Radiation Oncology Biology Physics, vol. 70, no. 3, pp. 685-692, 2008.

[26] U. Ricardi, A. R. Filippi, A. Guarneri et al., "Stereotactic body radiation therapy for early stage non-small cell lung cancer: results of a prospective trial," Lung Cancer, vol. 68, no. 1, pp. 72$77,2010$.

[27] T. Xia, H. Li, Q. Sun et al., "Promising clinical outcome of stereotactic body radiation therapy for patients with inoperable Stage I/II non-small-cell lung cancer," International Journal of Radiation Oncology Biology Physics, vol. 66, no. 1, pp. 117-125, 2006.

[28] F. Baba, Y. Shibamoto, H. Ogino et al., "Clinical outcomes of stereotactic body radiotherapy for stage I non-small cell lung cancer using different doses depending on tumor size," Radiation Oncology, vol. 5, no. 1, article 81, 2010.

[29] A. Takeda, N. Sanuki, E. Kunieda et al., "Stereotactic body radiotherapy for primary lung cancer at a dose of 50 Gy total in five fractions to the periphery of the planning target volume calculated using a superposition algorithm," International Journal of Radiation Oncology Biology Physics, vol. 73, no. 2, pp. 442448, 2009.

[30] A. W. Y. Ng, S. Y. Tung, and V. Y. W. Wong, "Hypofractionated stereotactic radiotherapy for medically inoperable stage I nonsmall cell lung cancer: report on clinical outcome and dose to critical organs," Radiotherapy and Oncology, vol. 87, no. 1, pp. 24-28, 2008.

[31] N. Andratschke, F. Zimmermann, E. Boehm et al., "Stereotactic radiotherapy of histologically proven inoperable stage I nonsmall cell lung cancer: patterns of failure," Radiotherapy and Oncology, vol. 101, no. 2, pp. 245-249, 2011.

[32] K. L. Stephans, T. Djemil, C. A. Reddy et al., "A comparison of two stereotactic body radiation fractionation schedules for medically inoperable stage I non-small cell lung cancer: the 
Cleveland clinic experience," Journal of Thoracic Oncology, vol. 4, no. 8, pp. 976-982, 2009.

[33] T. D. Crabtree, C. E. Denlinger, B. F. Meyers et al., "Stereotactic body radiation therapy versus surgical resection for stage I nonsmall cell lung cancer," Journal of Thoracic and Cardiovascular Surgery, vol. 140, no. 2, pp. 377-386, 2010.

[34] R. Onimaru, H. Shirato, S. Shimizu et al., "Tolerance of organs at risk in small-volume, hypofractionated, image-guided radiotherapy for primary and metastatic lung cancers," International Journal of Radiation Oncology Biology Physics, vol. 56, no. 1, pp. 126-135, 2003.

[35] S. Bral, T. Gevaert, N. Linthout et al., "Prospective, risk-adapted strategy of stereotactic body radiotherapy for early-stage nonsmall-cell lung cancer: results of a phase II trial," International Journal of Radiation Oncology Biology Physics, vol. 80, no. 5, pp. 1343-1349, 2011.

[36] N. C. van der Voort van Zyp, J.-B. Prévost, M. S. Hoogeman et al., "Stereotactic radiotherapy with real-time tumor tracking for non-small cell lung cancer: clinical outcome," Radiotherapy and Oncology, vol. 91, no. 3, pp. 296-300, 2009.

[37] M. Guckenberger, J. Wulf, G. Mueller et al., "Dose-response relationship for image-guided stereotactic body radiotherapy of pulmonary tumors: relevance of $4 \mathrm{D}$ dose calculation," International Journal of Radiation Oncology Biology Physics, vol. 74, no. 1, pp. 47-54, 2009.

[38] M. Turzer, O. T. Brustugun, E. Waldeland, and S. Helland, "Stereotactic body radiation therapy is effective and safe in patients with early-stage non-small cell lung cancer with low performance status and severe comorbidity," Case Reports in Oncology, vol. 4, no. 1, pp. 25-34, 2011.

[39] H. Onishi, K. Kuriyama, T. Komiyama et al., "Clinical outcomes of stereotactic radiotherapy for stage I non-small cell lung cancer using a novel irradiation technique: patient self-controlled breath-hold and beam switching using a combination of linear accelerator and CT scanner," Lung Cancer, vol. 45, no. 1, pp. 45$55,2004$.

[40] R. Timmerman, L. Papiez, R. McGarry et al., "Extracranial stereotactic radioablation: results of a phase I study in medically inoperable stage I non-small cell lung cancer," Chest, vol. 124, no. 5, pp. 1946-1955, 2003.

[41] R. Miralbell, S. A. Roberts, E. Zubizarreta, and J. H. Hendry, "Dose-fractionation sensitivity of prostate cancer deduced from radiotherapy outcomes of 5,969 patients in seven international institutional datasets: $\alpha / \beta=1.4(0.9-2.2)$ Gy," International Journal of Radiation Oncology Biology Physics, vol. 82, no. 1, pp. e17-e24, 2012.

[42] X. S. Qi, J. White, and X. A. Li, "Is $\alpha / \beta$ for breast cancer really low?" Radiotherapy and Oncology, vol. 100, no. 2, pp. 282-288, 2011.

[43] C. W. Song, L. C. Cho, J. Yuan, K. E. Dusenbery, R. J. Griffin, and S. H. Levitt, "Radiobiology of stereotactic body radiation therapy/stereotactic radiosurgery and the linear-quadratic model," International Journal of Radiation Oncology, vol. 87, pp. 18-19, 2013.

[44] M. Stuschke and C. Pöttgen, "Altered fractionation schemes in radiotherapy," Frontiers in Radiation Oncology, vol. 42, pp. 150156, 2010.

[45] M. Partridge, M. Ramos, A. Sardaro, and M. Brada, "Dose escalation for non-small cell lung cancer: analysis and modelling of published literature," Radiotherapy and Oncology, vol. 99, no. 1, pp. 6-11, 2011. 


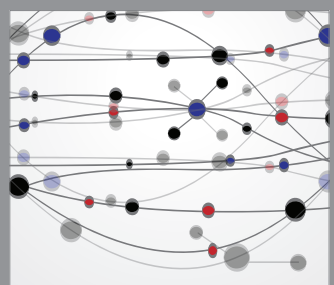

The Scientific World Journal
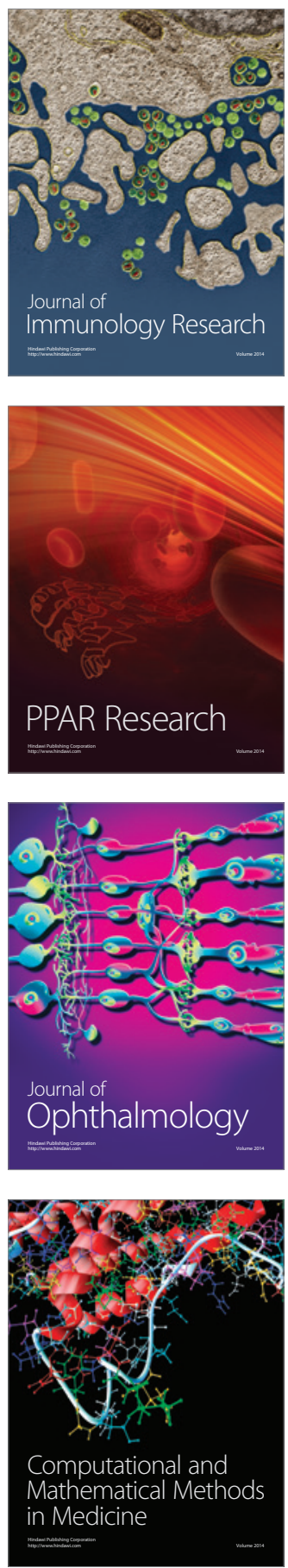

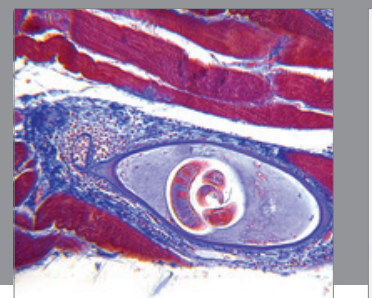

Gastroenterology

Research and Practice
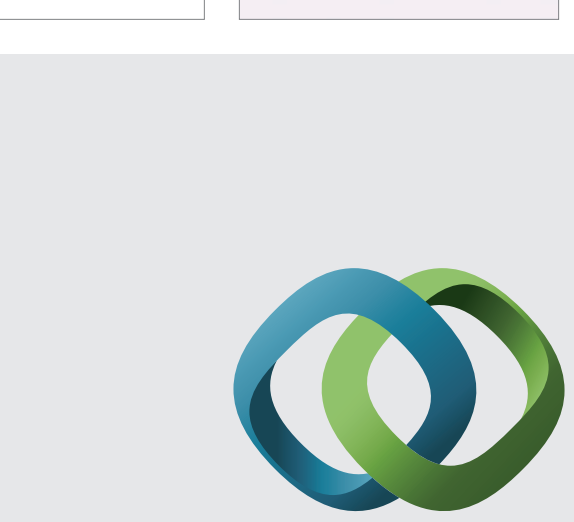

\section{Hindawi}

Submit your manuscripts at

http://www.hindawi.com
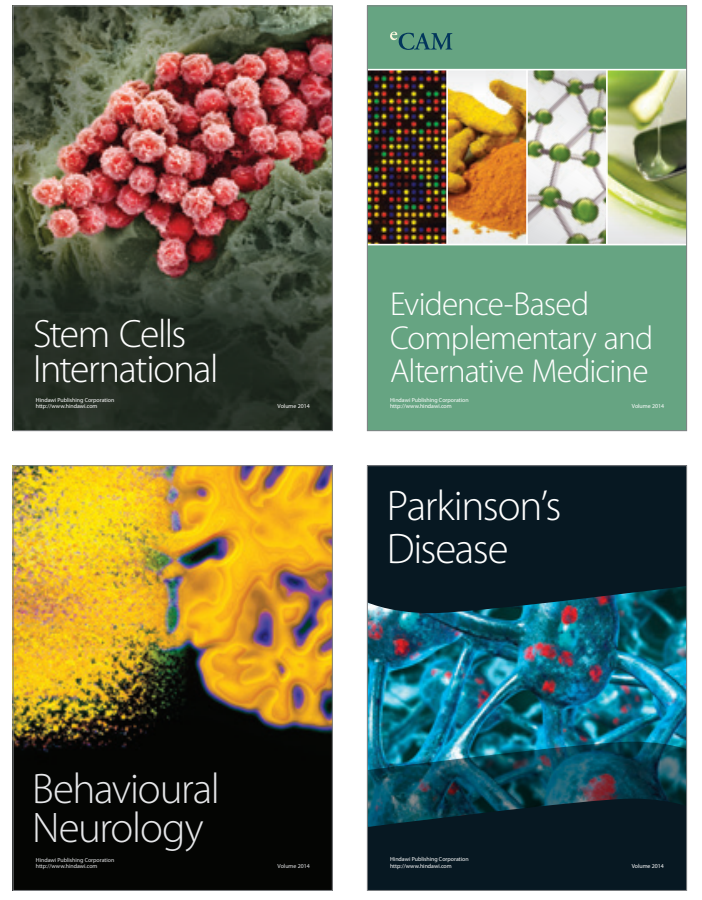
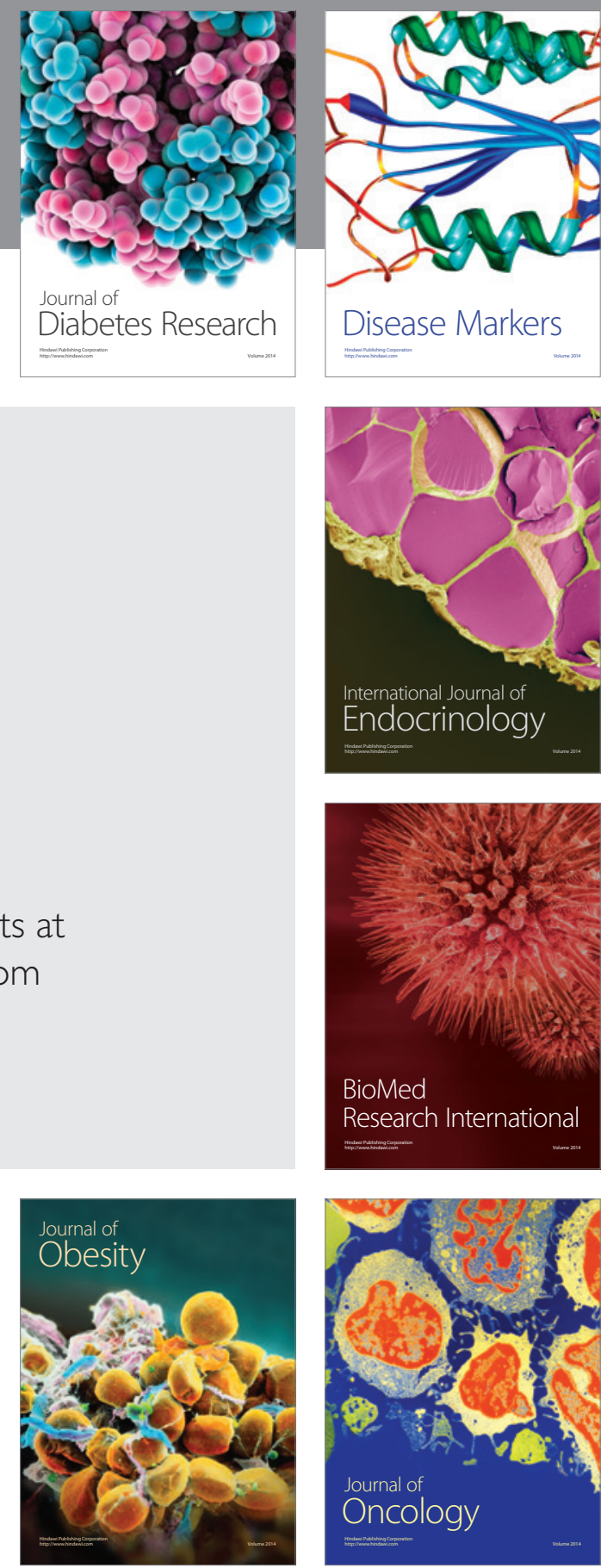

Disease Markers
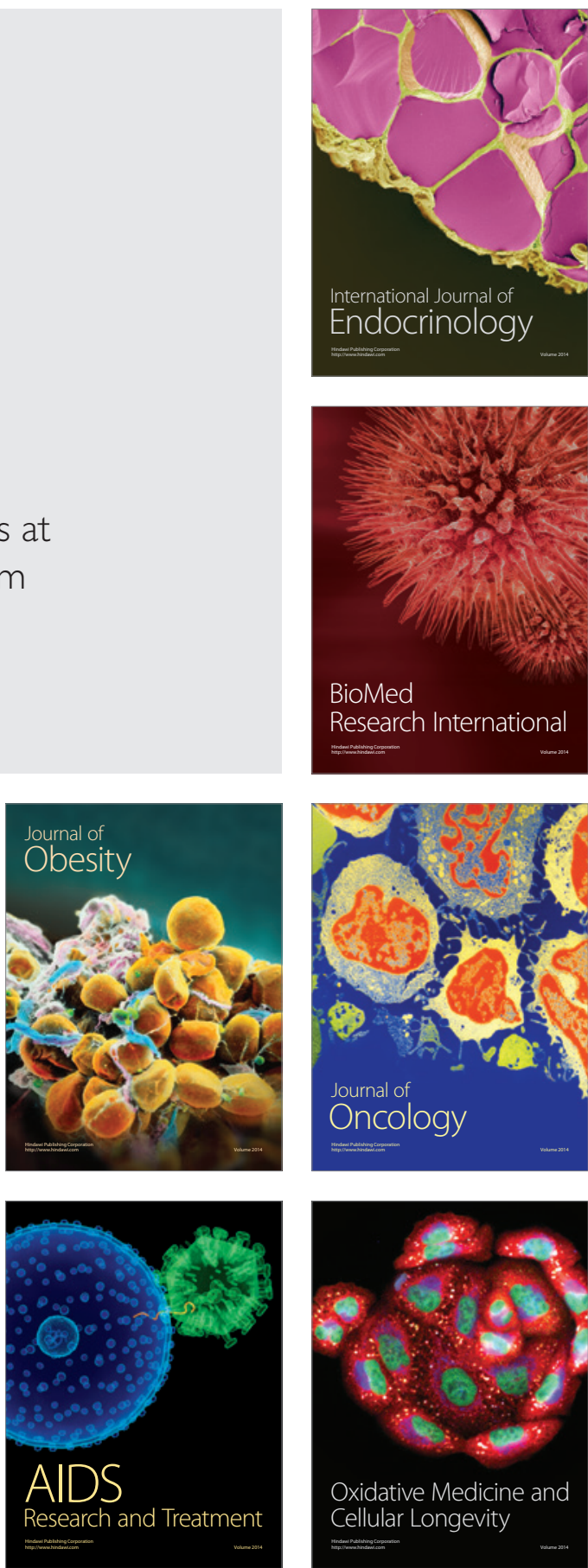